\title{
Adjuvant treatment of anogenital warts with systemic interferon: a systematic review and meta-analysis
}

\author{
Lukas Westfechtel, Ricardo Niklas Werner, Corinna Dressler, Matthew Gaskins, \\ Alexander Nast
}

- Additional material is published online only. To view please visit the journal online (http://dx.doi.org/10.1136/ sextrans-2017-053150)

Department of Dermatology, Venereology and Allergology, Division of Evidence-Based Medicine (dEBM), Charité Universitätsmedizin Berlin, Berlin, Germany

\section{Correspondence to} Lukas Westfechtel, Department of Dermatology, Venereology and Allergology, Division of Evidence-Based Medicine (dEBM), Charité Universitätsmedizin Berlin, Berlin 10117, Germany; lukas. westfechtel@gmx.net

Received 10 February 2017 Accepted 9 July 2017 Published Online First 17 August 2017

\section{CrossMark}

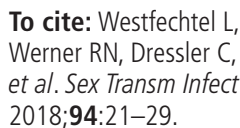

\begin{abstract}
Background Interferons are natural messenger

proteins that are used to treat various disease

entities. Due to their immunomodulating, antiviral and antiproliferative effects, the systemic administration of interferons after ablative treatment for anogenital warts (AGWs) has been advocated to increase clearance and decrease recurrence rates. However, studies investigating the efficacy of adjuvant systemic interferon have yielded inconsistent results. The objective of this systematic review and meta-analysis was to comprehensively assess and evaluate the available evidence from randomised controlled trials.

Methods A literature search was conducted in Cochrane Central Register of Controlled Trials, Embase and MEDLINE. Available data were classified according to the interferon type and dosage. Pooled effect estimates were calculated for predefined outcomes. The Cochrane Collaboration's risk of bias tool was used to assess the included trials and the Grading of Recommendations Assessment, Development and Evaluation (GRADE) approach to evaluate our confidence in the effect estimates.
\end{abstract}

Results Twelve trials were identified that met the inclusion criteria and assessed immunocompetent patients with external AGW. Compared with placebo, adjuvant alpha-, beta- and gamma-interferon were generally not significantly superior in terms of complete clearance over the short, intermediate or long term, nor with regard to intermediate- or long-term recurrence. However, the low-dose subgroup of adjuvant alphainterferon was significantly superior compared with placebo regarding intermediate-term complete clearance and recurrence. Further data were available for the comparison of different dosages of alpha- and betainterferon and for comparisons of the three interferon types. No significant differences were seen in these comparisons regarding efficacy. Data on quality of life were not available.

Conclusions The GRADE quality of the evidence ranged from 'very low' to 'high'. The significantly higher efficacy of low-dose alpha-interferon compared with placebo was based on a single trial, and our confidence in the effect estimates rated as 'low'. Overall, we found no reliable evidence favouring the systemic use of interferon after ablative treatment of AGW.

\section{INTRODUCTION}

Anogenital warts (AGWs, condylomata acuminata) are caused by infections with particular types of human papillomaviruses (HPVs). The main cause is HPV types 6 and 11, which are responsible for about $90 \%$ of all AGWs. ${ }^{\mathrm{w} 1}$ Since these viruses are predominantly sexually transmitted and highly contagious, the main risk factors are related to sexual activity such as the number of lifetime sexual partners and less frequent condom use. ${ }^{\mathrm{w} 23}$ The highest incidence rates have been reported for young women (aged 20-24) and men (aged 25-29) with peak rates of up to 8.61 cases per year in 1000 young Australian women. ${ }^{\text {w4 }}$ AGWs are widespread within the sexually active population and one of the most common sexually transmitted diseases.

Although AGWs are usually not referred to as a serious condition, the loss in quality of life (QoL) of patients with AGW may be significant. ${ }^{\text {w6-10 }}$ Besides physical symptoms such as pruritus and pain, the impact on sexuality and feelings of shame, guilt and anxiety are major limitations to QoL. ${ }^{\text {w11- }}$ 14 Currently available treatment options exhibit significant proportions of failures and recurrences (eg, podophyllotoxin solution 0.5\%: clearance 45\%-83\%, recurrence 13\%-100\%; electrosurgery: clearance $94 \%-100 \%$, recurrence $22 \%)$. ${ }^{\text {w15 }}$ This is especially true for immunocompromised patients. ${ }^{\text {w16 }}$ Identifying effective treatment strategies is of major importance.

Three different types of human interferon (IFN) are widely used for the treatment of various diseases due to their immunomodulating, antiviral and antiproliferative properties. Numerous studies have been conducted to investigate the topical, intralesional and systemic use of IFN as a treatment for AGW. ${ }^{\text {w17-21 }}$ Although some trials reported a significant superiority compared with placebo, clearing rates were not comparable to commonly used preparations (eg, imiquimod 5\% cream and podophyllotoxin $0.5 \%$ solution) or ablative treatment modalities (eg, laser therapy and electrosurgery). ${ }^{\text {w15 }}$ ${ }^{17}$ However, the systemic administration of IFN as an adjuvant therapy consecutive to an ablative treatment is still being discussed as a potential option to increase clearing rates and reduce recurrence rates. ${ }^{\text {w22 }}$ Some studies addressing the use of IFN as an adjuvant treatment detected a beneficial effect, ${ }^{1-4}$ whereas other trials found no significant differences to placebo. ${ }^{5-11}$ To address these ambiguities, we carried out a systematic review and meta-analysis summarising and evaluating the available evidence from randomised controlled trials (RCTs). 


\section{METHODS*}

We conducted a systematic review and meta-analysis following the methodology of the 'Cochrane Handbook for Systematic Reviews of Interventions" ${ }^{\text {, }}$ and the 'Grading of Recommendations Assessment, Development and Evaluation' (GRADE) approach. ${ }^{13}$ The eligibility criteria, searched databases and methods applied during the analysis were predefined in an internal protocol.

\section{Eligibility criteria}

Studies reporting on both immunocompetent and immunocompromised patients clinically diagnosed with internal (eg, vaginal, anal) or external (eg, penile, vulval, perianal) AGW were eligible. Exclusion criteria were cervical or intraurethral lesions and subclinical HPV infections.

All types (alpha, beta, gamma) of systemically administered IFN in combination with ablative (eg, laser, electrocautery, cryotherapy) or topical treatments (eg, podophyllotoxin solution, imiquimod cream) were eligible as intervention. The following treatment modalities could serve as comparator: placebo (or no treatment), a different IFN type or a different IFN dose, if combined with the same additional therapy as investigated in the interventional group.

Studies had to report at least one of the following outcomes:

- Complete clearance (CC) at 4 weeks ( \pm 4 weeks) after end of treatment (EOT) ('short-term CC')

- CC at 16 weeks ( \pm 8 weeks) after EOT ('intermediate-term CC')

- Dropouts due to adverse events (AEs).

Further outcomes were evaluated if available:

- $\mathrm{AE}$ (fever or flu-like symptoms, headache, fatigue)

- QoL (validated assessment tools)

- Recurrence of lesions at 16 weeks ( \pm 8 weeks) after EOT in patients who had a CC at 4 weeks ( \pm 4 weeks) after EOT ('intermediate-term recurrence')

- CC at 12 months ( \pm 6 months) after EOT ('long-term CC')

- Recurrence of lesions at 12 month ( \pm 6 months) after EOT in patients who had a CC at 4 weeks ( \pm 4 weeks) after EOT ('long-term recurrence').

Only randomised controlled studies with at least 10 participants in every group at baseline were eligible. Abstracts were excluded. The language was restricted to English and German.

\section{Literature searches and data management}

A comprehensive literature search was performed in the Cochrane Central Register of Controlled Trials, Embase and MEDLINE including MEDLINE in process. For the complete search strategy, see the online supplementary material 1.1. References of included articles were cross-checked for studies matching the inclusion criteria.

Two investigators (LW and RNW) independently screened titles and abstracts of the publications identified during the literature search. During the full-text evaluation, performed by the same two authors, reasons for the exclusion of studies were recorded.

Data extraction was conducted independently by two investigators (LW and RNW) using standardised data extraction forms. Disagreements concerning the inclusion of studies and extracted data were resolved through discussion. If this was not possible, a third investigator $(\mathrm{CD} / \mathrm{AN})$ was involved. Extracted data items are listed in the online supplementary material 1.2. For the meta-analyses of CC rates, intention-to-treat data were used. If these were not reported, non-responder imputation was applied.
The results of the meta-analyses were reported as risk ratios (RRs) with 95\% confidence intervals (CIs). ${ }^{14}$ Effect estimates were calculated with Mantel-Haenszel meta-analyses, applying a random-effects model. ${ }^{12}$ Pooling included different IFN subtypes (eg, alpha-2a, alpha-2b) but not different IFN types (alpha, beta, gamma). The available data were grouped according to the administered dose:

- Low dose (below 9 million International Units (MIU) per week)

- High dose (at least 9 MIU per week in at least 1 week during treatment)

- Very high dose (at least 18 MIU per week in at least 1 week during treatment).

When the IFN dose was individualised according to the participants' body surface area, the average of $1.79 \mathrm{~m}^{2}$ for adults was used to categorise the respective study. ${ }^{15}$ An overall effect estimate of a particular IFN type, comprising data of all the available dosing subgroups, was calculated in case of absence of statistical ( $\left.\mathrm{I}^{2} \geq 60 \%\right)$ and clinical heterogeneity. Review Manager, V.5.3.5, was used for all analyses. ${ }^{16}$

\section{Risk of bias and overall quality of evidence}

The GRADE approach was applied to assess the quality of the evidence. ${ }^{13}$ First, the risk of bias of each study was evaluated using the 'Cochrane Collaboration's tool for assessing risk of bias in randomised trials'. ${ }^{17}$ Subsequently, the overall confidence in the pooled effect estimates was evaluated at the outcome level. ${ }^{18}$ During this assessment, the risk of bias within the contributing studies, ${ }^{19}$ inconsistency, ${ }^{20}$ indirectness, ${ }^{21}$ imprecision $^{22}$ and publication bias $^{23}$ was considered. The criteria for rating these items are given in the online supplementary material 1.3. Based on this evaluation, the overall quality of the evidence was categorised as very low, low, moderate or high.

\section{RESULTS}

The literature search was conducted on 28 April 2016 and yielded 579 hits. After removing 216 duplicates, 363 remained for evaluation. During the title and abstract screening, 329 references were excluded. Of the 34 articles screened in full text, 22 further articles were excluded, resulting in the inclusion of 12 studies in the quantitative analysis. No further studies were identified through a cross-check of the included studies' reference lists. Figure 1 (PRISMA flowchart) illustrates the flow of information during study selection. Reasons for the exclusion of each study during the full-text evaluation are listed in the online supplementary material 2.1.

The 12 included studies ${ }^{2-112425}$ comprised 1070 participants in the relevant study arms and reported on immunocompetent patients only. Studies reporting on HIV-positive or other immunocompromised patients were not available. Regarding the location of warts, four trials ${ }^{2} 689$ reported solely on external warts, whereas eight trials ${ }^{3-5} 710112425$ considered both external and internal warts without reporting them separately. However, in all of these trials, the majority of participants had external lesions. Data on the impact of the evaluated interventions on QoL were not available. For detailed information regarding the study and sample characteristics, see table 1 .

The risk of bias within the included studies was heterogeneous (see figure 2, risk of bias summary). Our evaluations regarding confidence in the effect estimates (GRADE quality of the evidence) are reported along with the outcome results.

RRs, CIs and GRADE evaluations for all outcomes and subgroups are given in the Summary of Findings tables (see online 


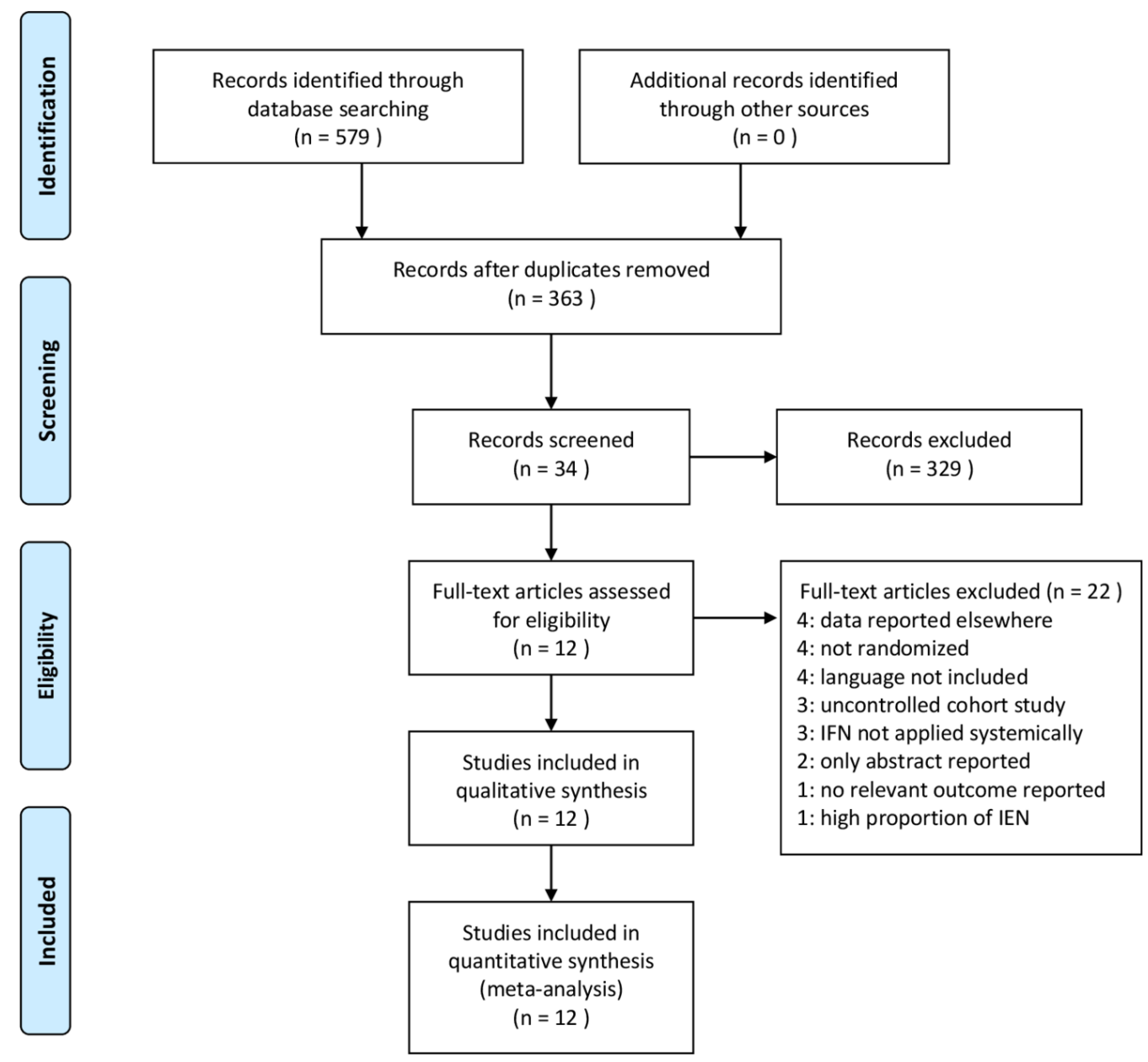

Figure 1 PRISMA flowchart. Flow of information during study selection. IEN, intraepithelial neoplasia; IFN, interferon.

supplementary material 2.4). For doses, treatment duration and other study characteristics, see table 1.

\section{Adjuvant alpha-IFN versus placebo or no treatment}

Eight trials ${ }^{3-10}(\mathrm{n}=757)$ compared alpha-IFN with placebo (or no treatment) combined with an additional treatment. Seven trials ${ }^{35-10}$ investigated high doses, and two trials ${ }^{46}$ investigated low doses of alpha-IFN. Regarding short-term CC, no significant differences between the groups were detected, neither in the overall pooled estimate, nor in the high-dose or low-dose subgroup (figure 3a). Data for intermediate-term CC showed no significant differences in the overall pooled estimate, as well as in the separate analysis of the high-dose subgroup. Within the low-dose subgroup, alpha-IFN was significantly superior to placebo (1 RCT, RR 3.09, 95\% CI 1.04 to 9.18 , GRADE: low) (figure $3 \mathrm{~b}$ ). No significant differences were detected regarding long-term CC (total: 3 RCTs, RR 1.00, 95\% CI 0.85 to 1.19 , GRADE: high, statistical heterogeneity: $\left.\mathrm{I}^{2}=0 \%\right)$. For intermediate-term recurrence, pooling of the highdose and low-dose subgroups was not appropriate due to statistically heterogeneity $\left(\mathrm{I}^{2}=84.5 \%\right)$; for the high-dose subgroup, no significant differences were detected, whereas the low-dose subgroup exhibited a significant superiority of adjuvant alpha-IFN compared with placebo (figure $3 \mathrm{c}$ ). No significant differences were detected regarding long-term recurrence (total: 3 RCTs, RR 1.00, 95\% CI 0.85 to 1.19 , GRADE: high, $\left.\mathrm{I}^{2}=0 \%\right)$. For dropouts due to $\mathrm{AE}$, data were available only for the high-dose regimen (5 RCTs, RR 2.57, 95\% CI 0.65 to 10.09, GRADE: moderate). Fever or flu-like symptoms were reported in a significantly higher frequency in participants receiving alpha-IFN injections, whereas no significant differences were detected for headache and fatigue. All AE data refer to the high-dose regimen (see online supplementary material 2.4.1).

\section{Adjuvant beta-IFN versus placebo or no treatment}

Two trials $(n=137)$ investigated adjuvant beta-IFN in a highdose $^{5}$ and very high dose $e^{2}$ combined with an ablative therapy. No significant differences between the verum and placebo groups were seen for short-term CC (1 RCT, RR 0.88, 95\% CI 0.45 to 1.73, GRADE: low) and intermediate-term CC (1 RCT, RR 1.22 , 95\% CI 0.95 to 1.56 , GRADE: low), both of these referring to a very high dose regimen. The RR regarding dropout due to AE could not be calculated due to zero events. ${ }^{5}$ Occurrence of AE showed no significant differences to placebo (see online supplementary material 2.4.2); these data refer to the high-dose regimen.

\section{Adjuvant gamma-IFN versus placebo or no treatment}

Two trials $(n=95)$ evaluated a placebo comparison of high-dose ${ }^{5}$ or low-dose ${ }^{11}$ gamma-IFN combined with ablative therapy. No significant differences were detected regarding long-term CC (1 RCT, RR 1.25 , 95\% CI 0.47 to 3.33, GRADE: moderate), intermediate-term recurrence (1 RCT, RR 1.20, 95\% CI 0.54 to 2.67, GRADE: moderate) and long-term recurrence (1 RCT, RR 1.00, 95\% CI 0.39 to 2.53 , GRADE: moderate); these data refer to the low-dose regimen. Regarding dropouts due to $\mathrm{AE}$, zero events 

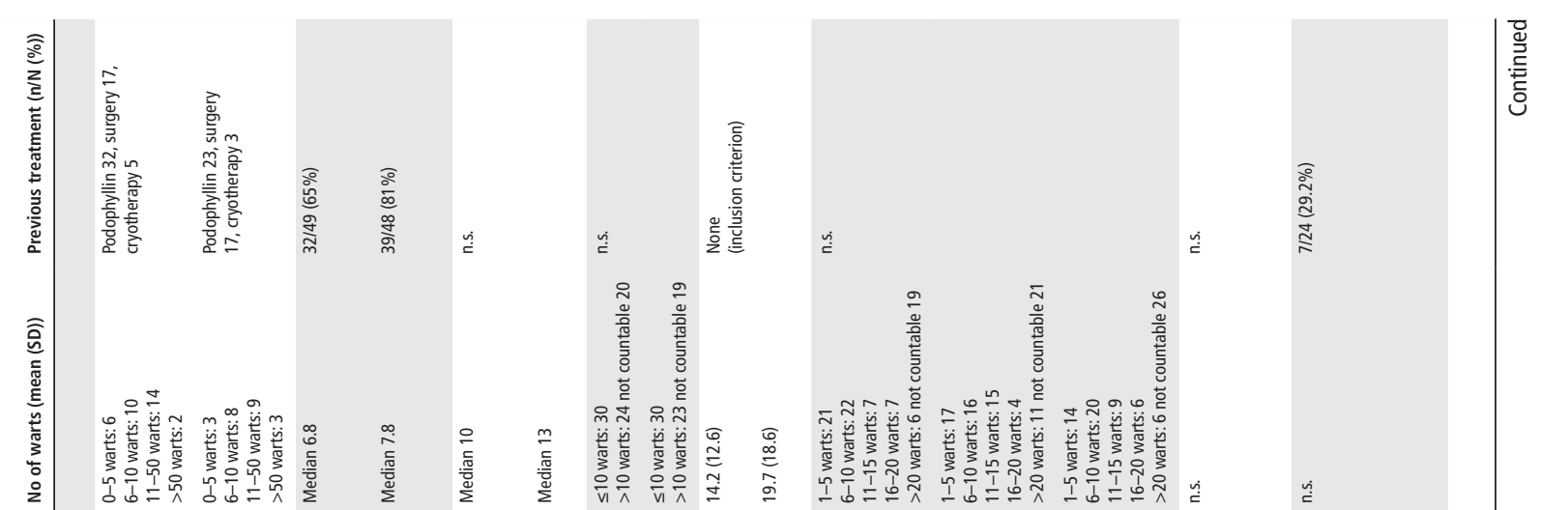

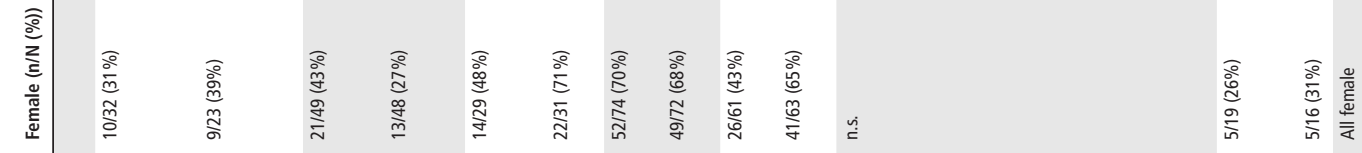

产

(1)

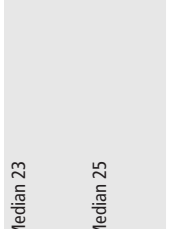

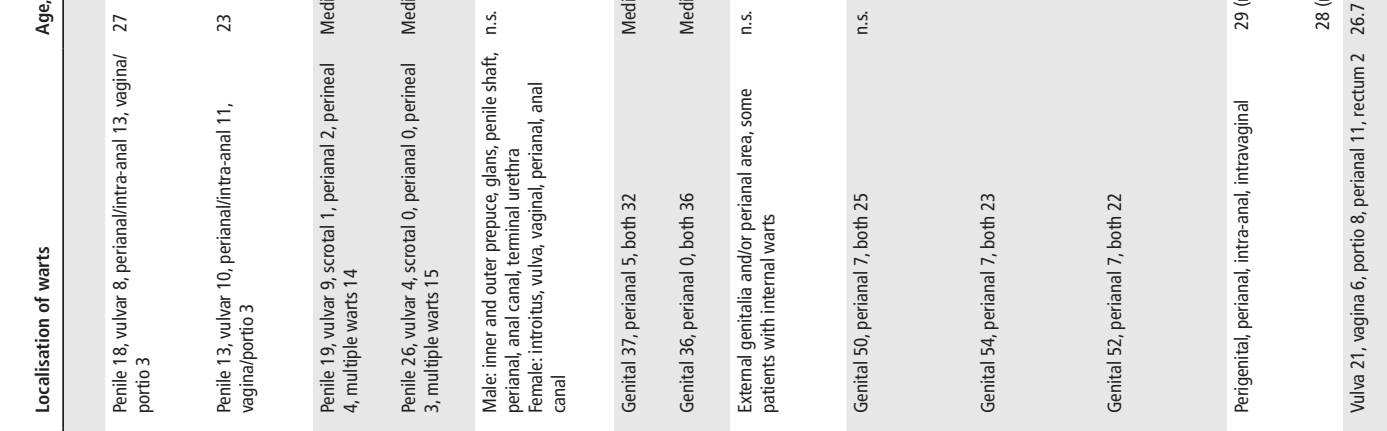

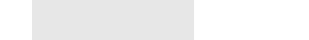$$
\text { (n) }
$$

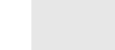

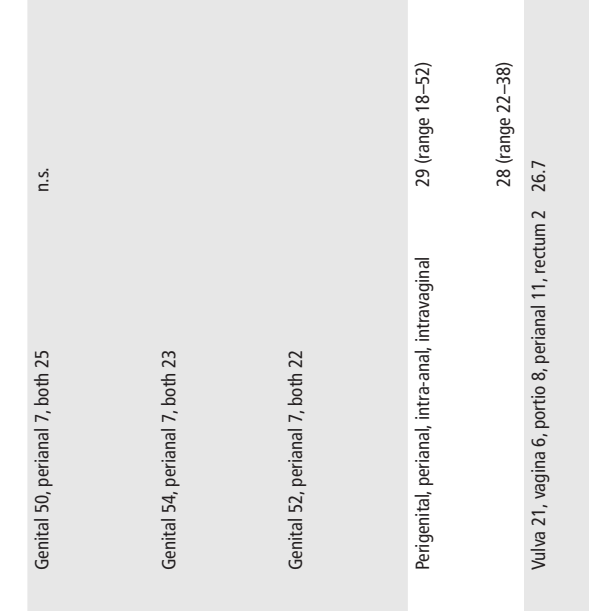

$\approx$

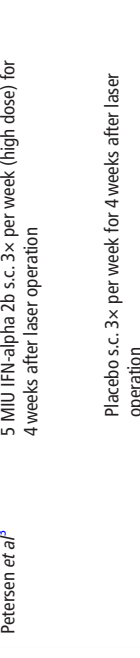
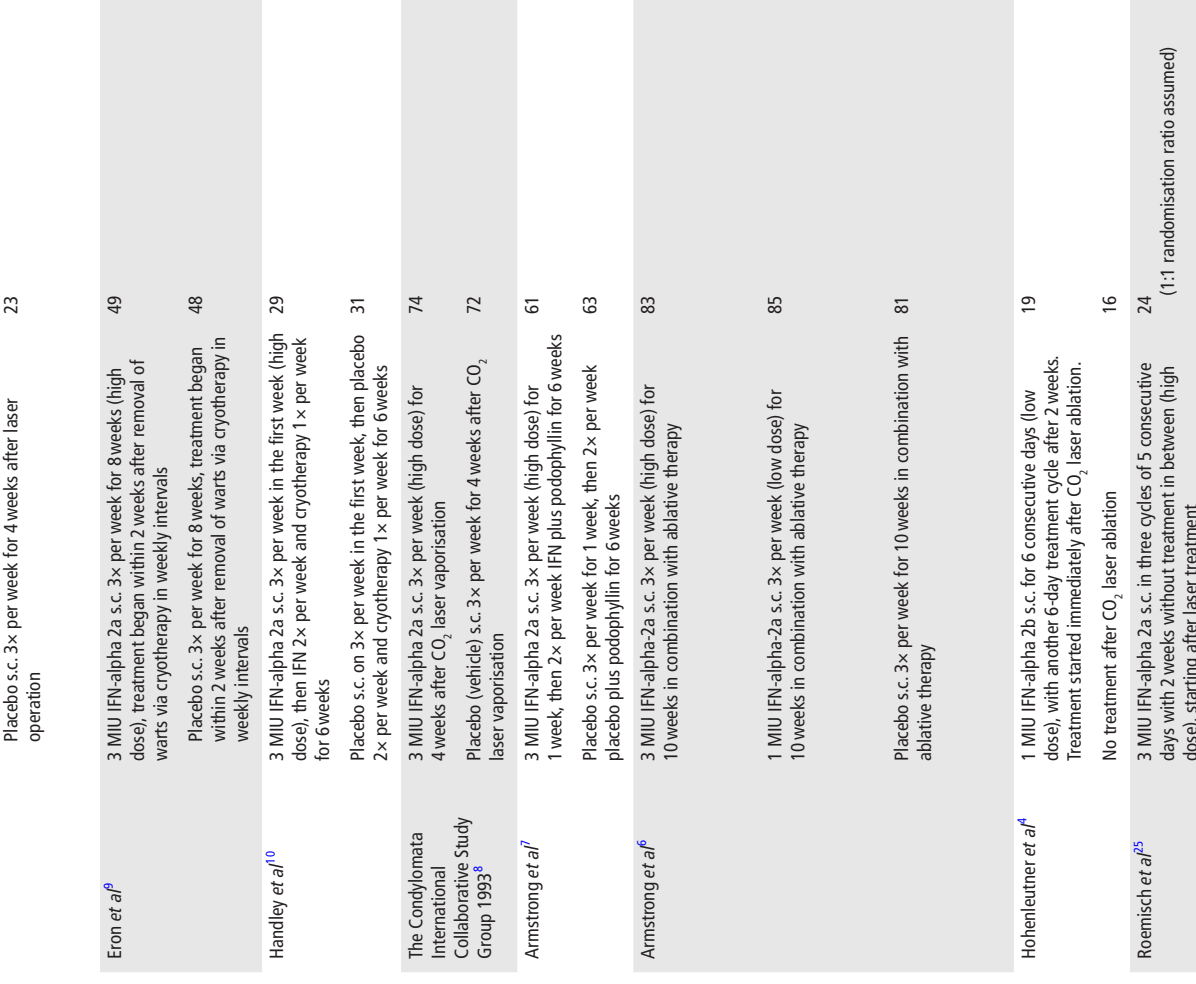


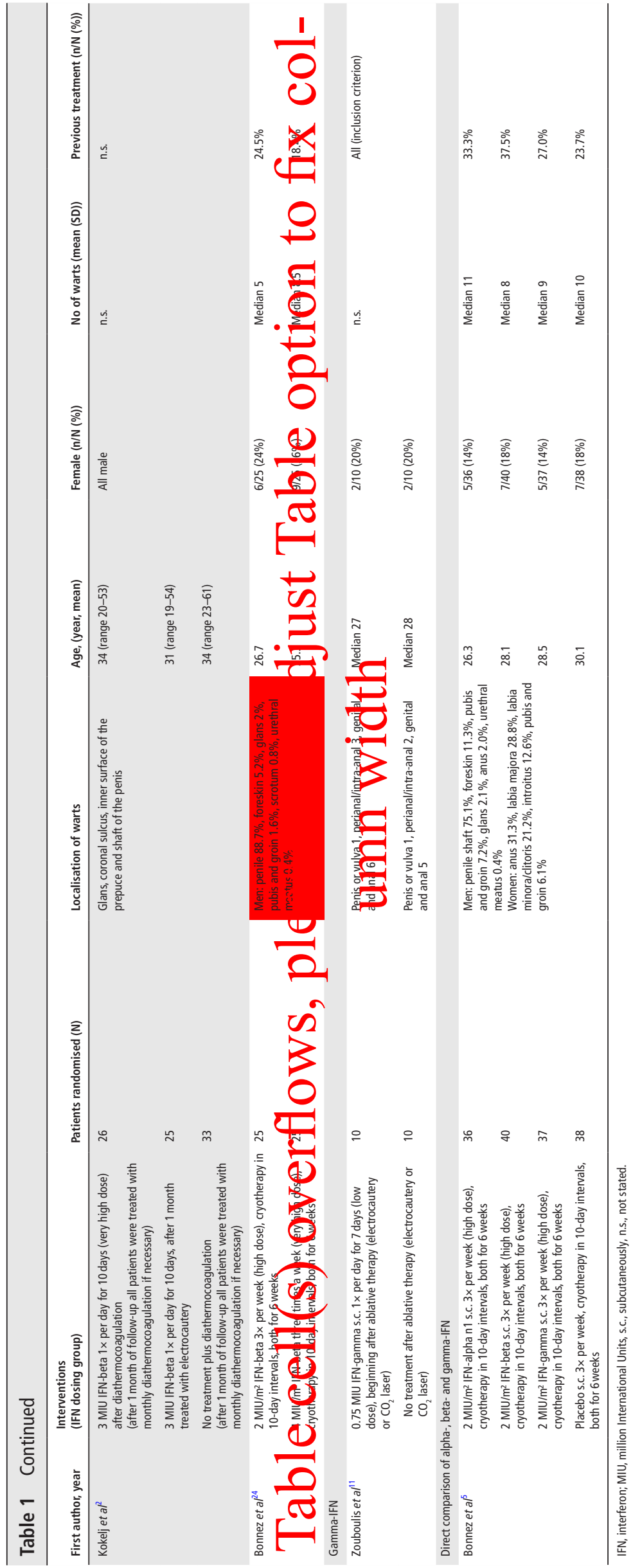

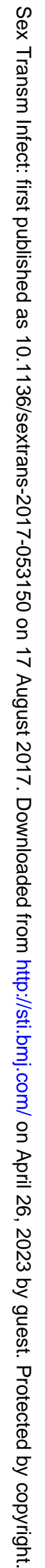




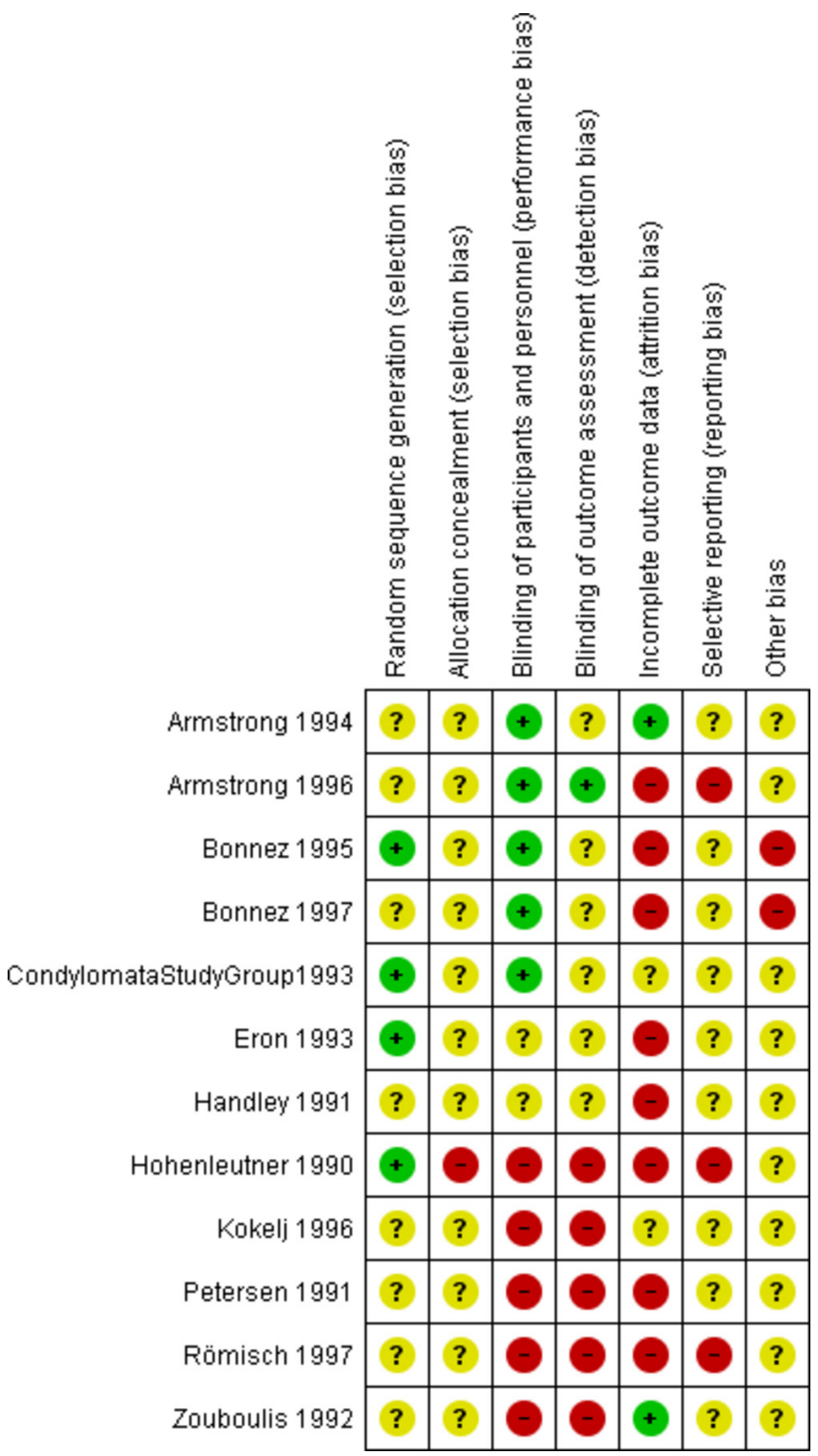

Figure 2 Risk of bias summary. Review authors' judgements about each risk of bias item for included studies.

occurred in both trials. AEs were reported in a higher frequency within the gamma-IFN group; a significant level was reached for fever or flu-like symptoms and headache but not for fatigue (see online supplementary material 2.4.3). AE data refer to the high-dose regimen.
Adjuvant alpha-IFN high dose versus low dose

Two studies, ${ }^{625}$ including 192 participants, evaluated a comparison of different alpha-IFN doses in combination with ablative therapy. Data were available for short-term CC, long-term CC, long-term recurrence and dropouts due to AE. No significant 


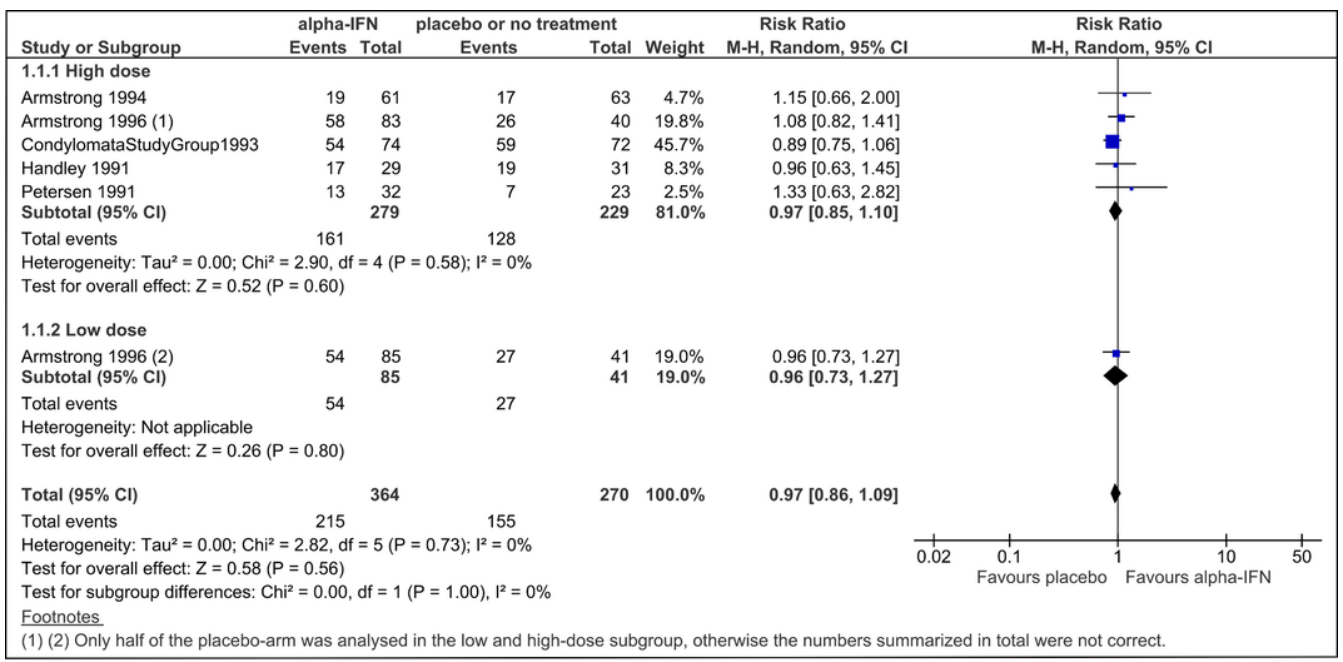

(1) (2) Only half of the placebo-arm was analysed in the low and high-dose subgroup, otherwise the numbers summarized in total were not correct.

Figure 3a: Adjuvant alpha-IFN vs. placebo, short-term CC. GRADE quality of the evidence: high dose subgroup: moderate; low dose subgroup: low; total effect estimate: moderate.

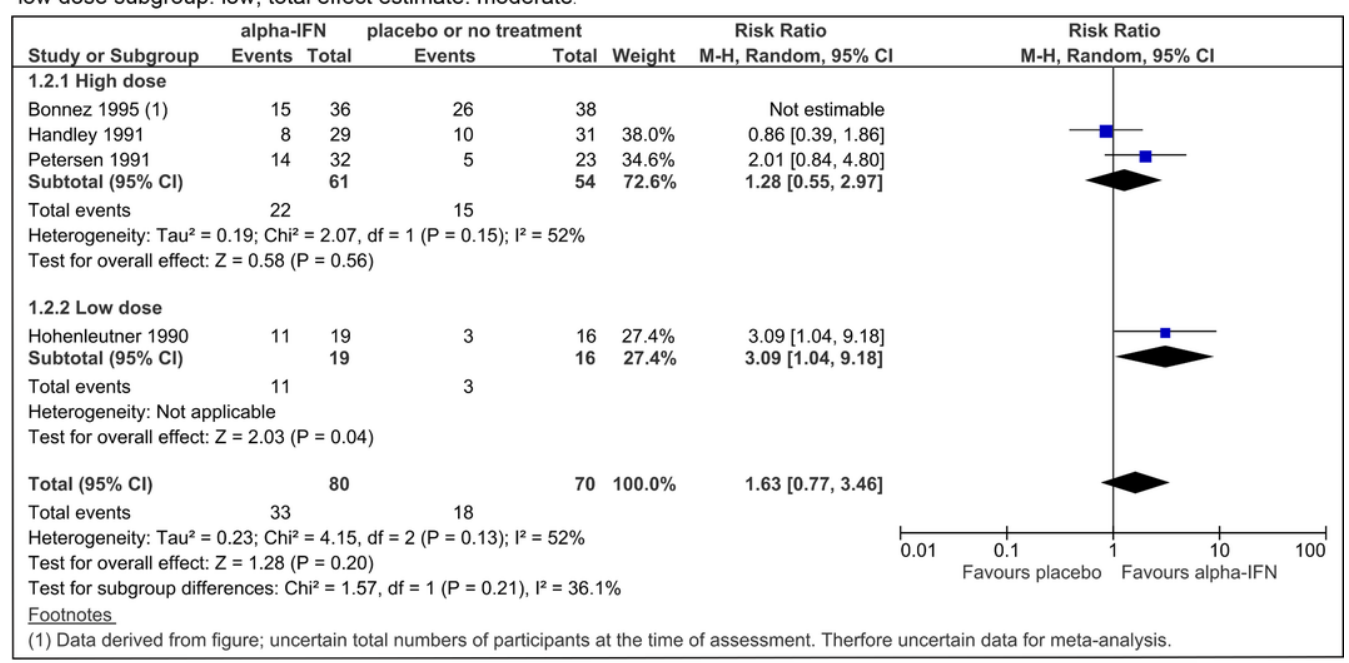

Figure 3a: Adjuvant alpha-IFN vs. placebo, intermediate-term CC. GRADE quality of the evidence: high dose subgroup: very low low dose subgroup: low; total effect estimate: very low.

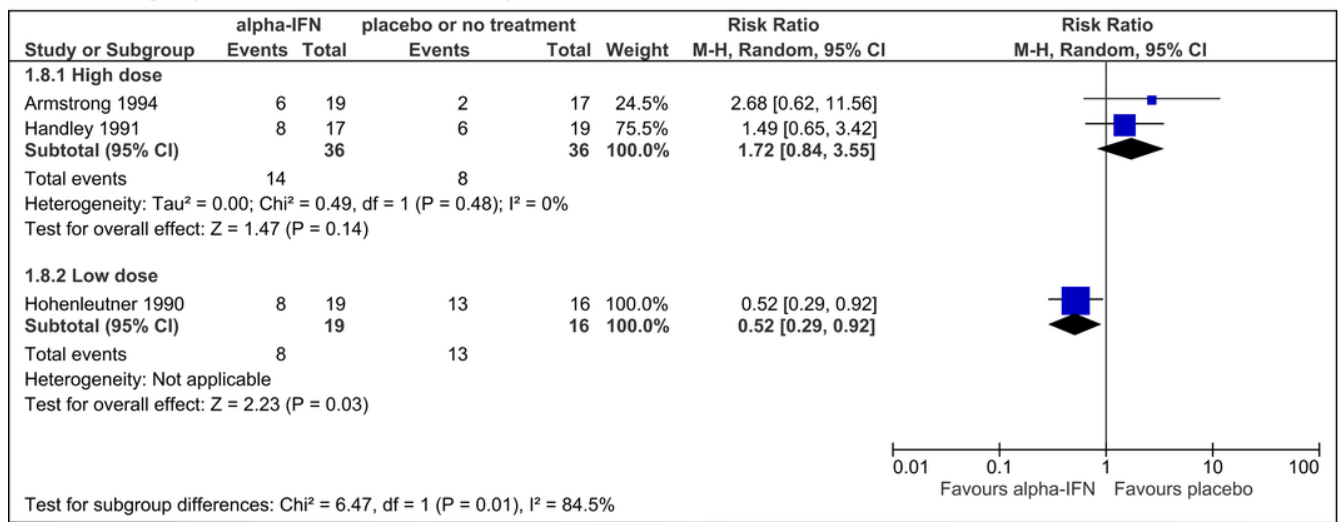

Figure 3a: Adjuvant alpha-IFN vs. placebo, intermediate-term recurrence. GRADE quality of the evidence: high dose subgroup: moderate; low dose subgroup: low; total effect estimate not calculated due to statistical heterogeneity.

Figure 3 Forest plots for efficacy outcomes of the comparison of adjuvant alpha-IFN versus placebo.

differences were found between the groups (see online supplementary material 2.4.4).

\section{Adjuvant beta-IFN very high dose versus high dose}

One $\operatorname{study}^{24}(n=50)$ compared different doses of beta-IFN in combination with cryotherapy. Data were available for intermediate-term CC, dropouts due to AE, fever or flu-like symptoms and headache. No significant differences were found between the groups (see online supplementary material 2.4.5).

\section{Direct comparisons of different IFN types}

The study by Bonnez and colleagues ${ }^{5}$ compared high-dose regimens of adjuvant alpha-, beta- and gamma-IFN, combined with cryotherapy in each of the groups. Results for efficacy outcomes 
were not extractable, since no absolute data were stated in this study (see online supplementary material 2.3). However, the authors reported no significant differences between the three types of IFN (page 1084). No significant differences were seen regarding dropouts due to $\mathrm{AE}$. Regarding $\mathrm{AE}$, the following results were seen:

- Alpha-IFN versus beta-IFN: AE occurred more often in the alpha-IFN group; however, differences were only significant regarding fever or flu-like symptoms.

- Beta-IFN versus gamma-IFN: participants receiving beta-IFN experienced fewer AE; these differences were only significant regarding fever or flu-like symptoms and headache.

- Alpha-IFN versus gamma-IFN: A general tendency was not noticed; with respect to headache, participants of the alpha-IFN group experienced significantly more events (see online supplementary material 2.4.6-2.4.8).

\section{Additional analysis}

Different subtypes of IFN (eg, alpha-2a and alpha-2b), as well as different types of combined therapies (eg, laser therapy and cryotherapy), were investigated in the included studies. These differences raise the question of whether or not the pooling of these studies is appropriate. We investigated the influence of these treatment modalities within the high-dose subgroup of the comparison adjuvant alpha-IFN versus placebo. Regarding this group, the highest number of studies was available, and variation in dose and dosing schedule was negligible (see table 1). The studies were allocated to subgroups regarding combined therapy (laser therapy vs cryotherapy vs podophyllin) and checked for subgroup differences. No significant differences were seen between these subgroups (eg, short-term-CC: $\chi^{2}=0.42, \mathrm{df}=2$, $\mathrm{p}=0.81$ ).

Additionally, we investigated the influence of different subtypes of alpha-IFN (alpha-2a vs alpha-2b vs alpha-n1) on the outcomes. No statistically significant subgroup differences were detected (eg, short-term-CC: $\chi^{2}=0.69, \mathrm{df}=1, \mathrm{p}=0.41$ ).

\section{DISCUSSION}

The present systematic review and meta-analysis summarised and evaluated the available evidence from RCTs addressing the adjuvant use of systemic IFN for AGW. The majority of the included studies investigated the comparison of adjuvant alpha-IFN versus placebo following ablative treatment in each of the study groups. Considering the overall effect estimates, no significant differences were seen in efficacy outcomes. Statistically significant superiority over placebo was only seen regarding intermediate-term CC and intermediate-term recurrence for a low-dose regimen of alpha-IFN. However, our confidence in these effect estimates was rated as 'low'. This rating was based on the imprecision of the results (with a questionable clinical significance of the difference between the groups) and the high risk of bias in the small $\operatorname{trial}^{4}(\mathrm{n}=35)$ which was available for this comparison. Assuming a dose-response relationship and given the non-superiority of high-dose alpha-IFN regimens compared with placebo, the significant superiority of the low-dose alpha-IFN regimen over placebo is not plausible and should be interpreted with caution.

For the comparisons of the other IFN types with placebo, few trials were available, and regarding efficacy, beta- and gamma-IFN were not significantly superior compared with placebo. Conforming to these findings, neither comparisons between different dosing regimens of a particular IFN type nor the comparison of the different IFN types (study of Bonnez et al, ${ }^{5}$ page 1084) exhibited significant differences regarding efficacy outcomes.

None of the assessed AEs were significantly different for the comparison of beta-IFN with placebo. Significantly higher rates of fever or flu-like symptoms were seen in patients treated with alpha- or gamma-IFN compared with placebo. Participants receiving gamma-IFN experienced headache significantly more often than participants in the placebo group. In direct comparisons, patients treated with beta-IFN showed significantly lower rates of fever or flu-like symptoms compared with those treated with alpha- or gamma-IFN. Similarly, the beta-IFN group exhibited significantly lower rates of headache compared with the gamma-IFN group. Despite these significant results for $\mathrm{AE}$, no significant differences were detected regarding dropouts due to AE. Thus, we conclude that AEs of all IFN types were generally not of a severe nature and usually acceptable for the participants.

The GRADE quality of the evidence was heterogeneous, ranging from 'very low' to 'high'. These ratings express our confidence in the effect estimates and are therefore crucial when deducting conclusions from the results. However, these ratings do not consider the total number of participants of the pooled analyses, and statistical insignificance may also result from insufficient statistical power to detect differences; to keep this in mind is especially important when interpreting the non-significant results of this review.

Adjuvant treatment with IFN is particularly discussed for patients who experienced multiple recurrences. Only a single trial $^{11}$ addressed solely recurrent AGW. Furthermore, the absence of eligible studies regarding immunocompromised patients, in whom these conditions occur more frequently, has to be seen as a limitation.

In our additional analyses of high-dose alpha-IFN, different subtypes of alpha-IFN and different combined treatments did not influence the CC rates. Although most data were available for the high-dose alpha-IFN subgroup, the total number of eight contributing studies was still small. Due to the small number of studies, the test for subgroup differences is not highly sensitive. Therefore, it is conceivable that the effect of IFN is depending on the subtype (eg, alpha- $2 \mathrm{a}$ vs alpha- $2 \mathrm{~b}$ ) and the combined treatment (eg, laser therapy vs cryotherapy). Also, the different dosing schedules (three times weekly vs daily) may differ in CC rates.

This review considered the patient-reported outcomes of headache and fatigue. However, none of the included trials investigated the interventions' effect on health-related QoL. This represents an important limitation, since QoL data should have a strong influence on the determination of treatment strategies.

\section{Conclusions}

The 12 studies identified as part of this meta-analysis of the adjuvant use of systemic IFN for AGW included only immunocompetent patients who predominantly had external AGW. No RCTs including immunocompromised patients were available. The quality of the evidence of the pooled effect estimates was heterogeneous and ranged from 'very low' to 'high'. The only adjuvant IFN treatment that showed statistically significant superiority over placebo with respect to the selected efficacy outcomes was low-dose adjuvant alpha-IFN. The quality of the evidence for these results, however, was rated as 'low'. Overall, no reliable evidence was available to support the use of systemic IFN as an adjuvant treatment for AGW.

* This systematic review and meta-analysis is part of a threephase series of reviews on HPV-associated anogenital lesions in 


\section{Key messages}

- Available treatment options for anogenital warts (AGWs) are far from optimal and characterised by high proportions of treatment failures and recurrences.

- Systemic interferon is still being discussed as addition to ablative treatment to overcome unsatisfactory treatment results for AGW; however, clinical studies have yielded inconsistent results.

- Regarding efficacy outcomes, superiority over placebo was solely seen for low-dose alpha-interferon; however, these data are at high risk for bias.

- Overall, no reliable evidence was available to support the use of systemic interferon as an adjuvant treatment for AGW.

different patient populations and with a focus on different interventions. Due to the same methodology used for the conduct of the review, parts of the Methods section are identical, and similarities in the description of the results may occur (doi:10.1136/ sextrans-2016-052768; 10.1136/sextrans-2016-053035).

Contributors LW, RNW, CD and AN developed the research question and methods section. LW developed the search strategy and excluded doublets. LW and RNW selected eligible studies during the title/abstract screening and full-text evaluation extracted data from the included studies, appraised the quality of the evidence, entered and double-checked data for RevMan and analysed/interpreted the data. AN and CD were involved in cases of dissent with respect to extracted data/ evaluations and participated in analysing and interpreting the data. RNW, AN, CD and MG contributed to the final editing of the draft. LW developed the draft of the manuscript, coordinated contributions of the coauthors and compiled the final draft.

Competing interests The Division of Evidence Based Medicine (dEBM) received research grants from various entities. The conduct of the current review was not funded. Previously, the conduct of a systematic review and meta-analysis of topical interventions for anogenital warts in immunocompetent patients has been funded with an institutional research grant by Meda Pharma. The dEBM received research grants not associated with the current work by the Paul-Ehrlich-Gesellschaft für Chemotherapie e.V., European Academy of Dermatology and Venereology, Medigene, European Dermatology Forum, Pfizer, Merz, Deutsche Dermatologische Gesellschaft, Deutsche Gesellschaft für Allergologie und klinische Immunologie, Deutsche Dermatologische Akademie and GSK. The dEBM is currently involved in the development of a German clinical practice guideline on the management of human papillomavirus-associated anogenital lesions. AN is responsible for the grants given to dEBM. LW, RNW, CD and MG declare to have no further conflicts of interests. AN has received personal honoraria for educational activity with direct or indirect sponsoring from Novartis, Pfizer, Boehringer Ingelheim, Bayer Healthcare, Jansen and Meda.

Patient consent This was a systematic review and meta-analysis.

Provenance and peer review Not commissioned; externally peer reviewed.

(c) Article author(s) (or their employer(s) unless otherwise stated in the text of the article) 2018. All rights reserved. No commercial use is permitted unless otherwise expressly granted.

\section{REFERENCES}

1 Reid R, Greenberg MD, Pizzuti DJ, et al. Superficial laser vulvectomy. V. Surgical debulking is enhanced by adjuvant systemic interferon. Am J Obstet Gynecol 1992;166:815-20.
2 Kokelj F, Stinco G, Torsello P. Efficacy of intramuscular interferon- $\beta$ in reducing relapses in the treatment of male patients with condylomata acuminata. J Dermatolog Treat 1996;7:7-9.

3 Petersen CS, Bjerring $\mathrm{P}$, Larsen J, et al. Systemic interferon alpha-2b increases the cure rate in laser treated patients with multiple persistent genital warts: a placebocontrolled study. Genitourin Med 1991;67:99-102.

4 Hohenleutner U, Landthaler M, Braun-Falco O. Postoperative adjuvant therapy with interferon alfa-2B following laser surgery of condylomata acuminata. Hautarzt 1990;41:545-8.

5 Bonnez W, Oakes D, Bailey-Farchione A, et al. A randomized, double-blind, placebocontrolled trial of systemically administered interferon-alpha, -beta, or -gamma in combination with cryotherapy for the treatment of condyloma acuminatum. J Infect Dis 1995;171:1081-9.

6 Armstrong DK, Maw RD, Dinsmore WW, et al. Combined therapy trial with interferon alpha-2a and ablative therapy in the treatment of anogenital warts. Genitourin Med 1996;72:103-7.

7 Armstrong DK, Maw RD, Dinsmore WW, et al. A randomised, double-blind, parallel group study to compare subcutaneous interferon alpha-2a plus podophyllin with placebo plus podophyllin in the treatment of primary condylomata acuminata. Genitourin Med 1994;70:389-93.

8 The Condylomata International Collaborative Study Group. Randomized placebocontrolled double-blind combined therapy with laser surgery and systemic interferonalpha $2 \mathrm{a}$ in the treatment of anogenital condylomata acuminatum. The Condylomata International Collaborative Study Group. J Infect Dis 1993;167:824-9.

9 Eron LJ, Alder MB, O'Rourke JM, et al. Recurrence of condylomata acuminata following cryotherapy is not prevented by systemically administered interferon. Genitourin Med 1993;69:91-3.

10 Handley JM, Horner T, Maw RD, et al. Subcutaneous interferon alpha 2a combined with cryotherapy vs cryotherapy alone in the treatment of primary anogenital warts: a randomised observer blind placebo controlled study. Genitourin Med 1991;67:297-302.

11 Zouboulis CC, Büttner P, Orfanos CE. Systemic interferon gamma as adjuvant therapy for refractory anogenital warts: a randomized clinical trial and meta-analysis of the available data. Arch Dermatol 1992;128:1413-4

12 Higgins J, Green S, eds. Cochrane Handbook for Systematic Reviews of Interventions Version 5.1.0 (updated March 2011). The Cochrane Collaboration, 2011. http:// handbook.cochrane.org/.

13 Atkins D, Best D, Briss PA, et al. Grading quality of evidence and strength of recommendations. BMJ 2004:328:1490.

14 Guyatt GH, Oxman AD, Santesso N, et al. GRADE guidelines: 12. preparing summary of findings tables-binary outcomes. I Clin Epidemiol 2013;66:158-72.

15 Sacco JJ, Botten J, Macbeth F, et al. The average body surface area of adult cancer patients in the UK: a multicentre retrospective study. PLoS One 2010;5:e8933.

16 Review Manager (RevMan) (Computer program) Version 5.3.5. Copenhagen: The Nordic Cochrane Centre, The Cochrane Collaboration, 2014.

17 Higgins JP, Altman DG, Gøtzsche PC, et al. The Cochrane collaboration's tool for assessing risk of bias in randomised trials. BMJ 2011;343:d5928.

18 Guyatt G, Oxman AD, Sultan S, et al. GRADE guidelines: 11. Making an overall rating of confidence in effect estimates for a single outcome and for all outcomes. J Clin Epidemiol 2013:66:151-7.

19 Guyatt GH, Oxman AD, Vist G, et al. GRADE guidelines: 4. Rating the quality of evidence-study limitations (risk of bias). J Clin Epidemiol 2011;64:407-15.

20 Guyatt GH, Oxman AD, Kunz R, et al. GRADE guidelines: 7. Rating the quality of evidence-inconsistency. J Clin Epidemiol 2011;64:1294-302.

21 Guyatt GH, Oxman AD, Kunz R, et al. GRADE guidelines: 8. Rating the quality of evidence -indirectness. J Clin Epidemiol 2011;64:1303-10.

22 Guyatt GH, Oxman AD, Kunz R, et al. GRADE guidelines 6 . Rating the quality of evidence-imprecision. J Clin Epidemiol 2011;64:1283-93.

23 Guyatt GH, Oxman AD, Montori V, et al. GRADE guidelines: 5. Rating the quality of evidence - publication bias. J Clin Epidemiol 2011;64:1277-82.

24 Bonnez W, Oakes D, Bailey-Farchione A, et al. A randomized, double-blind trial of parenteral low dose versus high dose interferon-beta in combination with cryotherapy for treatment of condyloma acuminatum. Antiviral Res 1997;35:41-52.

25 Roemisch M, Hillemanns P, Albrich W, et al. Adjuvant interferon and laser vapourisation in the treatment of condylomata acuminata—strategies to prevent recurrence. (German). Geburtshilfe Frauenheilkd 1997:57:141-7. 